Sari Yordi, MD

Tony and Leona Campane Center for Excellence for Image-Guided Surgery and Advanced Imaging Research, Department of Ophthalmology, Cole Eye Institute, Cleveland Clinic
Justis P. Ehlers, MD

Director, Tony and Leona Campane Center for Excellence

for Image-Guided Surgery and Advanced Imaging

Research, Department of Ophthalmology, Cole Eye Institute, Cleveland Clinic

\title{
COVID-19 and the eye
}

\section{Posted December 3, 2020}

\section{ABSTRACT}

As evidence on COVID-19 continues to emerge, its impact on the eyes is becoming more apparent, leading to a better understanding of ocular manifestations and transmission. This article details the recent information regarding COVID-19 from an ophthalmologic context, including prevalence, clinical features, impact on the clinic logistics, and key information for practitioners.

\section{INTRODUCTION}

The COVID-19 pandemic has created widespread challenges across healthcare with new data on the virus' effect on different organ systems emerging every day. In ophthalmology, there is an opportunity to explore the disease effects from a unique perspective with a potential to recognize the disease early and aid in its management. Several ophthalmic manifestations in patients with COVID-19 have been reported, with conjunctivitis-like symptoms being the most prevalent.

\section{PREVALENCE OF OPHTHALMIC SYMPTOMS}

Although literature on SARS-CoV-2 infection in ophthalmology is in its infancy, a growing number of studies are documenting the effect of the virus on the eye and surrounding structures. Several articles have presented evidence of ophthalmic manifestations in patients with COVID-19, with the most prevalent presentation being conjunctivitis-like symptoms. ${ }^{1-8}$ Interestingly, some cases reported that conjunctivitis was the initial presenting symptom. ${ }^{1,2}$ A large metaanalysis investigating the prevalence of ophthalmic disease found that in 2,228 patients diagnosed with

Disclaimer: The statements and opinions expressed in COVID-19 Curbside Consults are based on experience and the available literature as of the date posted. While we try to regularly update this content, any offered recommendations cannot be substituted for the clinical judgment of clinicians caring for individual patients.

doi:10.3949/ccjm.87a.ccc068
COVID-19, 95 (4.3\%) reported ocular symptoms and $21(0.9 \%)$ presented with ophthalmic symptoms as their initial complaint. ${ }^{2}$ A more recent meta-analysis of 12 studies in 1,930 subjects supported these findings reporting that conjunctivitis/conjunctival congestion presented in up to $8 \%$ of COVID-19 patients. $^{9}$

\section{OPHTHALMIC MANIFESTATIONS OF COVID-19}

Overall, the most common manifestations appear to be conjunctivitis or conjunctivitis-like features such as epiphora, hyperemia, and chemosis (ie, swelling of the conjunctiva). These symptoms have been noted in the early disease stages in outpatient settings or during hospital admission for severe disease. Nonspecific ophthalmic symptoms also have been reported, including photophobia, dry eye, foreign body sensation, and blurry vision. ${ }^{2,3}$ Currently, there are no clear distinguishing factors between COVID-19-related conjunctivitis and typical viral conjunctivitis, but that could change as more cases appear.

Other possible ophthalmic manifestations range from maculopathy ${ }^{4}$ to neuro-ophthalmic disease. ${ }^{10-12}$ Virgo and Mohamed described paracentral acute middle maculopathy in a patient with new onset scotoma following febrile illness, later confirmed by serology as caused by SARS-CoV-2 infection. ${ }^{4}$ Optical coherence tomography showed hyperreflective changes in the inner and outer plexiform layer with inner nuclear layer volume loss consistent with paracentral acute middle maculopathy. ${ }^{4}$ As with many of these early case reports, causality related to SARSCoV-2 infection compared with incidental association with COVID-19 status remains unclear. A list of ophthalmic conditions associated with COVID-19 can be seen in Table 1 .

Analyzing ophthalmic manifestations in COVID19 can be challenging. The novel nature of the virus, the apparent low prevalence of concurrent ophthalmic disease, its high infectivity, and poten- 
TABLE 1

\author{
Select ophthalmic conditions reported in patients \\ with COVID-19 \\ Conjunctivitis \\ Paracentral acute middle maculopathy ${ }^{4}$ \\ Acute macular neuroretinopathy ${ }^{4}$ \\ Miller Fisher syndrome ${ }^{10}$ \\ Polyneuritis cranialis ${ }^{10}$ \\ Panuveitis and optic neuritis" \\ Oculomotor nerve paresis ${ }^{12}$ \\ Nonspecific symptoms: Photophobia, dry eye, foreign body \\ sensation, blurry vision ${ }^{2}$
}

tially life-threatening complications all limit data acquisition and ancillary testing. Given the highly infectious nature of the virus, ancillary testing that is not crucial to disease management is often appropriately avoided; however, this limits data collection on ophthalmic features in patients with active COVID19 disease. Moreover, the ambiguity of ophthalmic symptoms and signs during COVID-19 could lead to misattribution of investigative findings. Therefore, it is essential to confirm potential positive findings whenever possible to limit uncertainty and lower the risk of misinterpretation.

\section{TRANSMISSION OF SARS-COV-2}

Studies on SARS-CoV-2 transmission are constantly being updated, but most sources agree that the majority of spread is attributed to respiratory droplets. Research has found that viral shedding of SARS-CoV-2 can remain positive for up to 35 days after symptom onset. ${ }^{13}$ However, current evidence on viral shedding in tears and conjunctival secretions is mixed. Several studies have tried to isolate the virus in tears and/or conjunctiva via reverse transcription polymerase chain reaction (RT-PCR). In a case series review of 43 patients, Karimi and colleagues attempted to isolate the virus in tears or conjunctiva using RT-PCR tests. ${ }^{14}$ Additionally, the authors analyzed results from 6 studies for possible correlations between positive results on nasopharyngeal swabs, sputum samples, and ocular swabs, and their relationship with documented ophthalmic symptoms $(\mathrm{N}=$ 338).

Their results showed SARS-CoV-2 in the tears of
$7 \%$ of patients with lab-confirmed or clinically diagnosed COVID-19 (3 of 43 patients). ${ }^{14}$ However, their pooled analysis of 6 studies showed detection rates ranging from $0 \%$ to $5 \%$. The disparity of results could be explained by differences in collection techniques, sampling timing, disease status and severity, and/ or viral quantities in collected samples. These studies also emphasized the lack of association between COVID-19-positive conjunctival RT-PCR results and the presence of conjunctival symptoms. At this time, it is unclear whether conjunctival secretions could be a mechanism of community spread. In addition, the systemic infectious capacity of viral exposure to the conjunctival surface remains unknown.

\section{MINIMIZING TRANSMISSION}

Given the close patient-doctor proximity during the ophthalmic examination, it is important to be vigilant regarding transmission risks. Aytogan and associates conducted a prospective study investigating the presence of SARS-CoV-2 on surfaces in the ophthalmology examination room. ${ }^{6}$ After sanitizing all surfaces and confirming negative RT-PCR results on 5 predefined zones, a total of 22 asymptomatic patients were examined along with 9 visitors. At the end of the day, the investigators found SARS-CoV-2 material in 2 samples taken from the zone within 1 meter of the patients' seat, specifically on the slit lamp breath shield and phoropter surface.

To minimize the infection risk of patients, visitors, and healthcare professionals, it is vital to institute appropriate clinical and surgical practices. The American Academy of Ophthalmologists has published a detailed breakdown of protocols for seeing patients that can maximize the safety of all involved parties. ${ }^{15}$ For routine appointments, major points include the following recommendations.

\section{Screening and social distancing}

- Prior to appointment entry, patients should be screened for COVID-19 symptoms, including recent history of fever, respiratory illness, loss of smell, or close contact with someone with confirmed COVID-19 in past 2 weeks. If any are present, infection control personnel and the local/ state health department should be notified.

- Maintain social distancing for patients in waiting rooms.

- Limit the number of visitors.

Personal protection

- Masks should be worn by patients and clinicians at all times. 
- Clinicians should wear eye protection whenever possible. If certain examinations require them removed, care must be taken to prevent contamination of medical devices.

Hand and surface hygiene

- Clinicians must thoroughly wash their hands before and after patient contact.

- Hand sanitizer should be readily available to patients, clinicians, and staff.

- Surfaces and equipment should be cleaned with virucidal wipes between each appointment.

If a patient has symptoms suggestive of COVID19 or confirmed infection, routine eye care should be postponed until after a quarantine and resolution of symptoms. Local- and facility-specific guidelines should be followed for both routine and urgent procedures. More information can be found on the American Academy of Ophthalmologists website. ${ }^{15}$

\section{PRIMARY CARE CONSIDERATIONS}

In primary care, management may vary per region/ hospital depending on COVID-19 prevalence and local guidelines for COVID-19-related illness. For patients with conjunctivitis symptoms and COVID19 , there are currently no established specific treatments for COVID-19-related conjunctivitis. Supportive care with artificial tears and cold compresses would be first-line therapies. A careful history and examination for associated ophthalmic symptoms, including visual acuity, that may merit consultation with ophthalmology, should be performed. Specific findings of concern include visual acuity loss and diplopia. As with other types of conjunctivitis, contact lens wearers should be warned about discontinuing contact lens wear until the condition has resolved. Disposable contact lenses that were worn when symptomatic should be discarded.

\section{CONCLUSION}

To minimize the infection risk of patients, visitors, and healthcare professionals, it is important to institute appropriate clinical and surgical practices. In ophthalmology, emerging data are revealing important insights into the impact of COVID-19 on the eyes. Additional research is needed to better understand potential effects of COVID-19 on ocular tissues and its potential manifestations. It is crucial to adopt safe operational practices to enable ongoing management of vision-threatening diseases in this patient population.

\section{DISCLOSURES}

The authors report no relevant financial relationships which, in the context of their contributions, could be perceived as a potential conflict of interest.

\section{REFERENCES}

1. Cheema M, Aghazadeh H, Nazarali S, et al. Keratoconjunctivitis as the initial medical presentation of the novel coronavirus disease 2019 (COVID-19). Can J Ophthalmol 2020; 55(4):e125-e129. doi:10.1016/j.jcjo.2020.03.003

2. Ho D, Low R, Tong L, Gupta V, Veeraraghavan A, Agrawal R. COVID19 and the ocular surface: a review of transmission and manifestations. Ocul Immunol Inflamm 2020; 28(5):726-734. doi:10.1080/092 73948.2020.1772313

3. American Academy of Ophthalmology. COVID-19 background primer for ophthalmologists. https://www.aao.org/headline/covid19-background-primer-ophthalmologists. Accessed November 24, 2020.

4. Virgo J, Mohamed M. Paracentral acute middle maculopathy and acute macular neuroretinopathy following SARS-CoV-2 infection. Eye (Lond) 2020; Jul 3:1-2. doi:10.1038/s41433-020-1069-8

5. Xia J, Tong J, Liu M, Shen Y, Guo D. Evaluation of coronavirus in tears and conjunctival secretions of patients with SARS-CoV-2 infection. J Med Virol 2020; 92(6):589-594. doi:10.1002/jmv.25725

6. Aytogan H, Ayintap E, Özkalay Yilmaz N. Detection of coronavirus disease 2019 viral material on environmental surfaces of an ophthalmology examination room. JAMA Ophthalmol 2020; 138(9):990-993. doi:10.1001/jamaophthalmol.2020.3154

7. Guan WJ, Ni ZY, Hu Y, et al; China Medical Treatment Expert Group for Covid-19. Clinical characteristics of coronavirus disease 2019 in China. N Engl J Med 2020; 382(18):1708-1720. doi: 10.1056/ NEJMoa2002032

8. Wu P, Duan F, Luo C, et al. Characteristics of ocular findings of patients with coronavirus disease 2019 (COVID-19) in Hubei Province, China. JAMA Ophthalmol 2020; 138(5):575-578. doi: 10.1001/ jamaophthalmol.2020.1291

9. Cao K, Kline B, Han Y, Ying GS, Wang NL. Current evidence of 2019 novel coronavirus disease (COVID-19) ocular transmission: a systematic review and meta-analysis. Biomed Res Int 2020; Oct 24:7605453. doi: 10.1155/2020/7605453

10. Gutiérrez-Ortiz C, Méndez-Guerrero A, Rodrigo-Rey S, et al. Miller Fisher syndrome and polyneuritis cranialis in COVID-19. Neurology 2020; 95(5):e601-e605. doi:10.1212/WNL.0000000000009619

11. Benito-Pascual B, Gegúndez JA, Díaz-Valle D, et al. Panuveitis and optic neuritis as a possible initial presentation of the novel coronavirus disease 2019 (COVID-19). Ocul Immunol Inflamm 2020; 28(6):922-925. doi:10.1080/09273948.2020.1792512

12. Dinkin M, Gao V, Kahan J, et al. COVID-19 presenting with ophthalmoparesis from cranial nerve palsy. Neurology 2020; 95(5):221-223. doi:10.1212/WNL.0000000000009700

13. Zhou F, Yu T, Du R, et al. Clinical course and risk factors for mortality of adult inpatients with COVID-19 in Wuhan, China: a retrospective cohort study. Lancet 2020; 395(10229):1054-1062. doi: 10.1016/ S0140-6736(20)30566-3

14. Karimi S, Arabi A, Shahraki T, Safi S. Detection of severe acute respiratory syndrome coronavirus- 2 in the tears of patients with coronavirus disease 2019. Eye 2020; 34(7):1220-1223. doi:10.1038/ s41433-020-0965-2

15. American Academy of Ophthalmology. Important coronavirus updates for ophthalmologists. https://www.aao.org/headline/alertimportant-coronavirus-context. Accessed November 24, 2020.

Correspondence: Justis P. Ehlers, MD, Department of Ophthalmology, Cole Eye Institute, i32, Cleveland Clinic, 9500 Euclid Avenue, Cleveland, OH 44195; ehlersj@ccf.org 